\title{
Correction: miR-200c inhibits TGF- $\beta$-induced-EMT to restore trastuzumab sensitivity by targeting ZEB1 and ZEB2 in gastric cancer
}

\author{
Xinliang Zhou - Xinyi Men · Riyang Zhao · Jing Han - Zhisong Fan · Yudong Wang • Yalei Lv • Jing Zuo • \\ Lianmei Zhao $\cdot$ Meixiang Sang $\cdot$ Xian-De Liu $\cdot$ Baoen Shan
}

Published online: 5 November 2020

(c) The Author(s), under exclusive licence to Springer Nature America, Inc. 2020

\section{Correction to: Cancer Gene Therapy \\ https://doi.org/10.1038/s41417-017-0005-y}

The original version of this Article contained inaccuracies in Figs. $2 \mathrm{a}$ and $4 \mathrm{~d}$ (one micrograph has been duplicated and presented as something else), Figs. $2 \mathrm{~b}$ and $4 \mathrm{c}$ (one micrograph has been duplicated and presented as something else) and Figs. $3 \mathrm{~b}$ and $4 \mathrm{j}$ (the two beta-actin bands are more similar than you would expect). All figures have been corrected in both the PDF and HTML versions of the Article.

Figure 4d: A large number of wound-healing assays graphs were taken, and had been taken as the cell control for the NCI-N87 (NCI-N87 and NCI-N87 NC), however there was negligence and repetition in the naming of the pictures. Therefore, these eventually led to the wrong insertion of the image when the authors prepared the figures. One image was repeated in Fig. 2a and Fig. 4d. Figure 4d has been corrected.

Figure 4c: Due to a large number of invasion assay photos taken and similarity of images, there was an error during image selection when the authors prepared the figure. The NCI-N87 results were used in NCI-N87-200c inhibitors group. One micrograph in Fig. 2c and Fig. $4 \mathrm{c}$ has been duplicated. Figure $4 \mathrm{c}$ has been corrected.

Figures $3 \mathrm{~b}$ and $4 \mathrm{j}$ : The two beta-actin bands in Fig. $3 \mathrm{~b}$ and Fig. $4 j$ were very similar. We provided the original image. The extreme similarity of the bands does raise doubts. Therefore, the authors have provided two other images and modified Fig. $3 \mathrm{~b}$ and Fig. $4 \mathrm{j}$ to eliminate these doubts.

The amendments made to the figures do not affect the conclusions of this research. 
Fig. 3
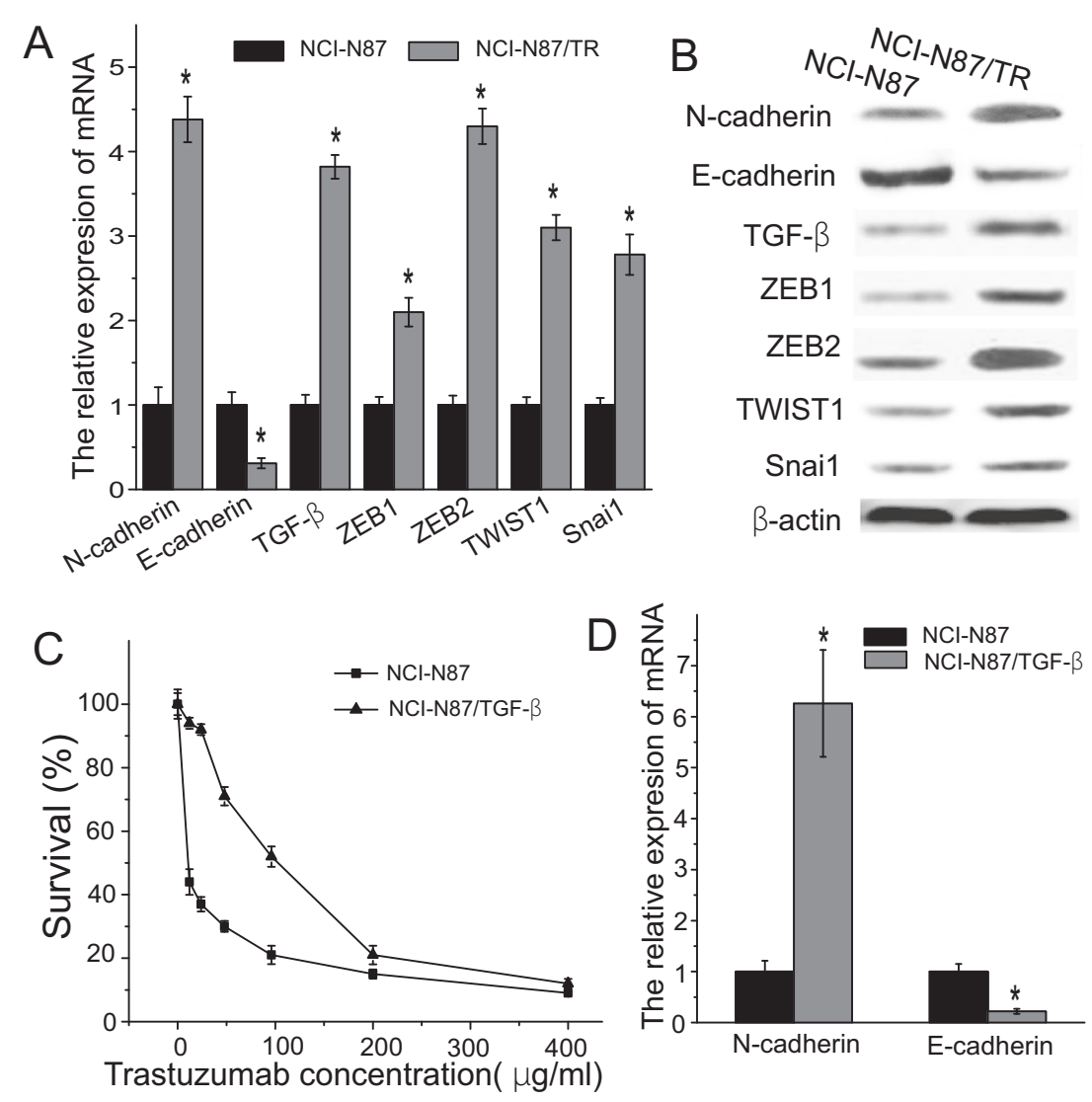


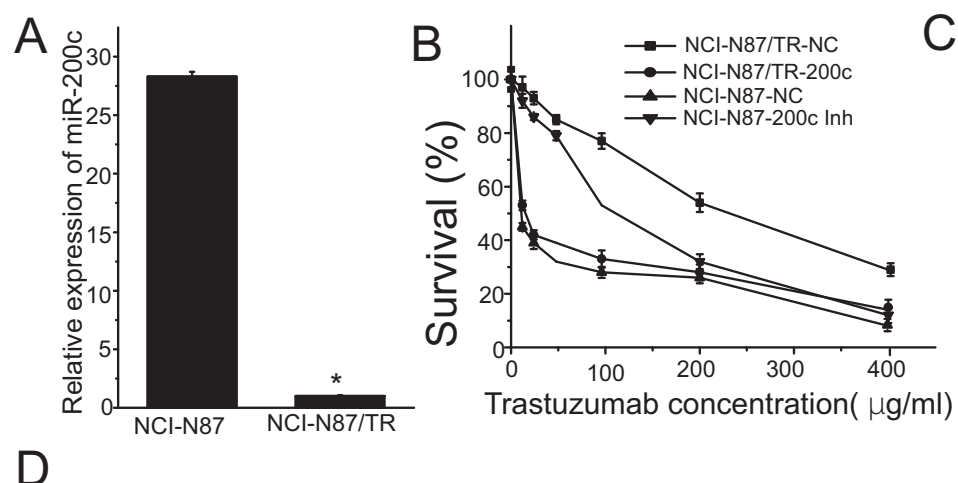

C NCI-N87/TR-NCI-N87/TR-200C

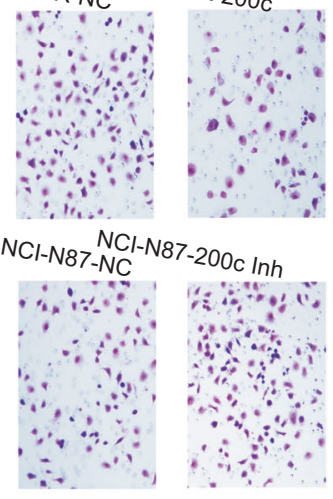

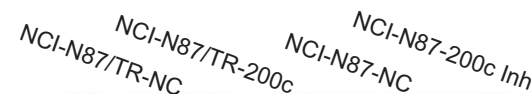

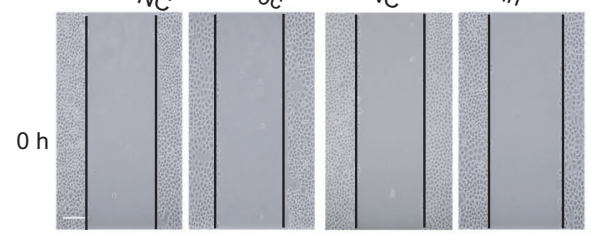

E
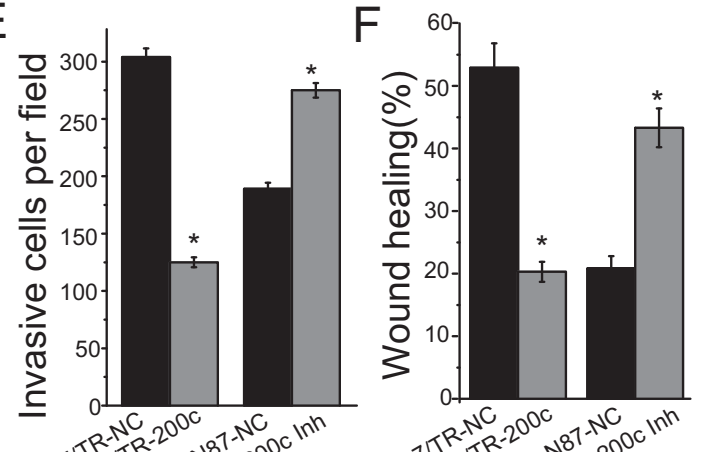

G

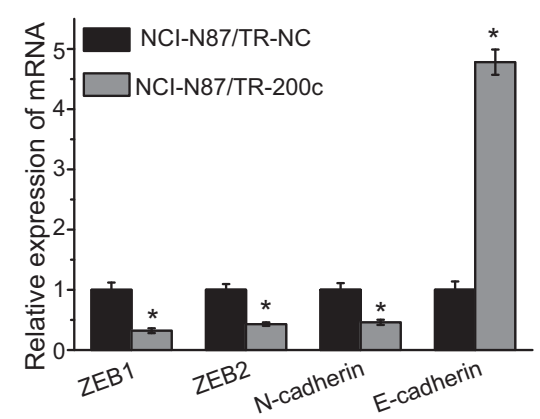

nothoting
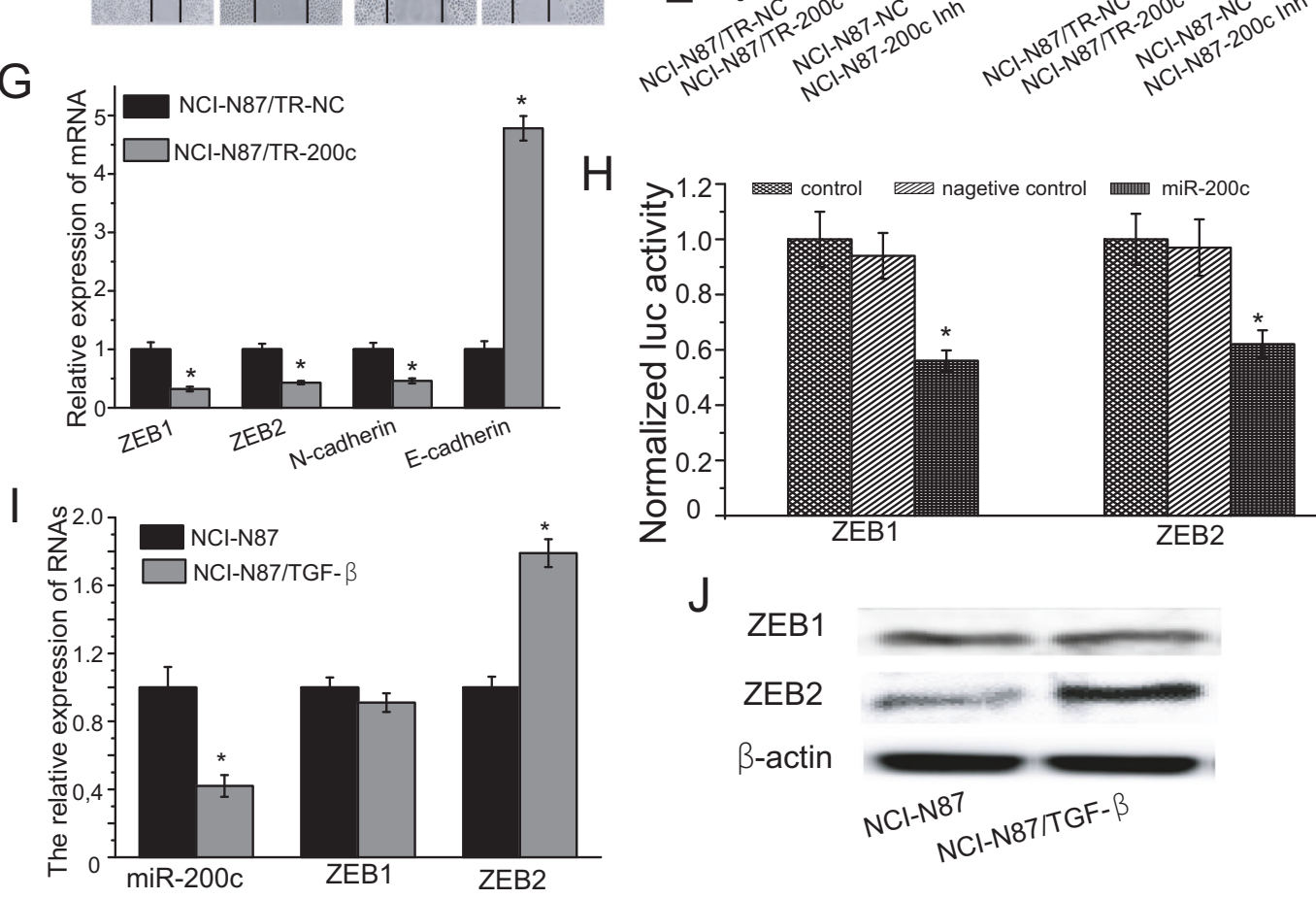

$\mathrm{H}$
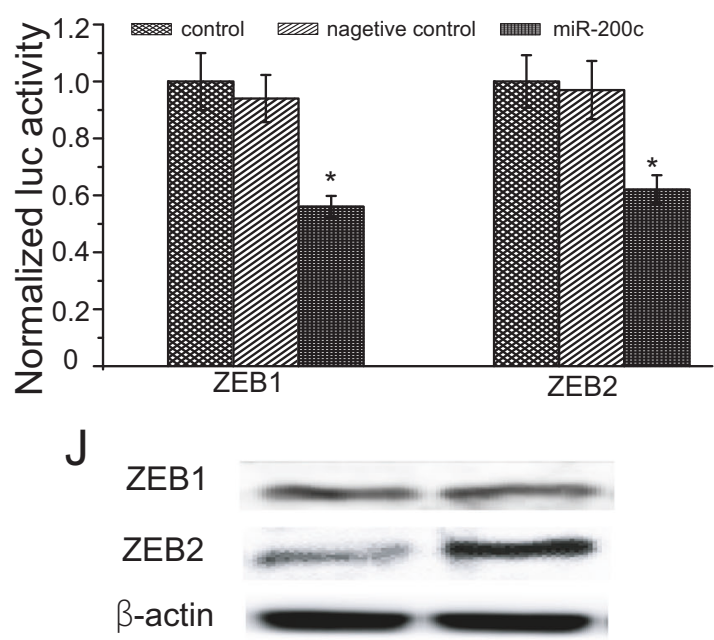

NCI-N87
NCI-N87/TGF- $\beta$

Fig. 4 